\section{Reporting guidelines: checklists for specific study designs}

\author{
Phil Wiffen
}

The growth of evidence-based medicine (EBM) has been followed by a flood of guidelines and advice on the reporting of specific study designs. This started with guidelines for randomised controlled trials and the CONSORT statement (this area seems to be plagued with acronyms). CONSORT stands for Consolidated Standards of Reporting Trials. They were developed in response to real problems in reporting where a trial may have been conducted to a very high standard and the related clinical trial reports may extend to hundreds of pages. In contrast, the publication of the trial in the medical literature is usually confined to some 3000 words. The temptation for authors of such papers is to build up the results section and minimise the methods in order to generate more interest and impact. The EBM world sees the methods section as key to assessing the quality of the research, and reporting guidelines were developed to cover all aspects of the report. The majority of the major medical journals have signed up to the use of such guidelines and that includes $E J H P^{1}$ The instructions for authors section on the EJHP website require the following five particular statements to be followed:

1. CONSORT statement-for randomised controlled trials

2. PRISMA statement-for systematic reviews

3. EVEREST statement-for economic evaluations

4. STARD statement-for diagnostic research papers

5. STROBE statement-for observational studies.

The reporting guideline community has organised itself under the acronym 'Equator'-Enhancing the QUAlity and Transparency Of health Research. ${ }^{2}$ The

Correspondence to Professor Phil Wiffen, Churchill Hospital, Pain Research Unit, Old Road, Oxford OX3 7LE, UK; phil.wiffen@ndcn.ox.ac.uk equator website lists the checklists and flow charts for a number of guidelines and has links to some 323 reporting guidelines, which in my view is verging on the ridiculous.

That said, the CONSORT, PRISMA and STROBE statements do lead to the improvement of higher quality papers, and authors of these types of reports are encouraged to use them in submissions to EJHP.

Arguably, the type of report that is most commonly poorly described in submissions to EJHP is the systematic review. In $E J H P$, we are keen to publish such reports as they can provide a valuable insight into an area of practice or treatment options. They sometimes provide a clear steer on what should be done or they may, equally valuably, highlight an area where little is known and ideally stimulate research into the topic. The PRISMA checklist ${ }^{3}$ contains 27 items and the PRISMA flow chart should be embedded in any report. Common problems in reporting systematic reviews include:

1. The objective. There needs to be a clear statement of the clinical question, which includes descriptions of the participants, interventions, comparisons, outcomes and study designs sought. Quite often, authors do not differentiate between high-quality and poor-quality designs like anecdotal reports.

2. Methods. Methods are a key part of any report as they enable an assessment of quality. Search strategies are often poorly described and often do not have the date of the last search. There is considerable confusion over databases such as PubMed as a resource and not infrequently this is the only source. PubMed is a useful resource for a quick look for information and can be compared in some ways to Google. It has all sorts of algorithms behind it that restrict what is presented. Ideally, systematic reviews should use Medline and other relevant databases. Pharmacy reviews should also search International Pharmaceutical Abstracts, but this is difficult to access and the search feature is not as well developed as other databases. Inclusion and exclusion criteria for study selection should be specified along with details of how results will be assessed and presented.

3. Results. These should include the results of the search strategy and the numbers of papers included and excluded (the flow diagram helps here). A summary table of the key points in each included paper can be helpful but may need to be published online if large. Results should be synthesised if appropriate and presented in a clear way.

4. Discussion. This is an opportunity to add a view on the importance of the results and also to suggest the implications for practitioners. A useful addition can be some suggestions about future research into the topic.

Reporting guidelines, if used carefully, make the preparation of reports an easier task as they provide a framework for the paper. In addition, they improve the chances of a paper reaching publication.

EAHP Statement 6: Education and Research

\section{Competing interests None.}

Provenance and peer review Commissioned; internally peer reviewed.

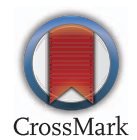

To cite Wiffen P. Eur J Hosp Pharm 2016;23:307.

Eur J Hosp Pharm 2016;23:307.

doi:10.1136/ejhpharm-2016-001115

\section{REFERENCES}

1 http://ejhp.bmj.com/site/about/guidelines.xhtml (accessed Sep 2016).

2 http://www.equator-network.org (accessed Sep 2016).

3 http://www.equator-network.org/reporting-guidelines/ prisma/ (accessed Sep 2016). 